\title{
An alternative to Unibase/glycol as an effective nonhydrating electrolyte medium for the measurement of electrodermal activity
}

\author{
SHARON L. DORMIRE ${ }^{\mathrm{a}}$ AND JANET S. CARPENTER ${ }^{\mathrm{b}}$

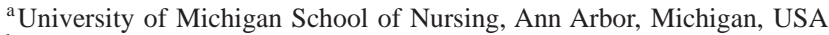 \\ b Vanderbilt University School of Nursing, Nashville, Tennessee, USA
}

\begin{abstract}
Although Unibase cream has been an effective medium for electrodermal recordings, its production has recently been discontinued. This study compared alternative media to Unibase for effectiveness. Three base creams similar to Unibase were initially compared for in vitro viscosity and effectiveness. Based upon this analysis one cream was eliminated. A volunteer sample of 6 postmenopausal women with hot flashes and 6 young women without flashes was recruited. Media were evaluated for viscosity and effectiveness of skin conductance. Results indicated that one electrolyte media produced readings within the normal range, identified true positive hot flashes, and produced few false negative readings whereas the second produced largely unreadable results with many false negative hot flashes. An appropriate substitute for Unibase as an effective electrolyte medium for measurement of electrodermal activity was identified.
\end{abstract}

Descriptors: Unibase, Velvachol, Hot flashes, Menopause, Skin conductance, Electrodermal electrolyte media

Schneider and Fowles (1978) found that polyethylene glycol and potassium chloride could be effectively combined with Unibase cream (Parke-Davis, Warner-Lambert, Morris Plains, NJ) to produce a suitably viscous cream that would provide effective recordings of electrodermal activity. This Unibase/glycol combination has been used extensively in objective monitoring of hot flashes via sternal skin conductance monitoring (Carpenter, Andrykowski, Freedman \& Munn, 1999; Freedman, 1989; Freedman, Norton, Woodward, \& Cornelissen, 1995) and a variety of other studies for the past 20 years. Recently, however, production has been discontinued of the Unibase cream needed to create the electrolyte cream developed by Schneider and Fowles. The purpose of this study was to determine whether any of three creams similar to Unibase in composition would function effectively as a medium for use on the human skin and provide optimal electrodermal recordings.

\section{Methods and Measures}

This collaborative study was conducted at two major universities in the southeastern United States. The researchers first consulted with university-based pharmacists to identify creams similar to Unibase in chemical composition and physical properties. Three creams were identified as being similar and thus were evaluated in this study: Hydrophilic ointment (E. Fougera and Co., Melville, NY), Aquabase (Paddock Laboratories Inc., Minneapolis, MN),

Address reprint requests to: Sharon Dormire, University of Michigan, 400 North Ingalls, Ann Arbor, MI 48109, USA. E-mail: Sdormire@ umich.edu. and Velvachol (Healthpoint, San Antonio, TX). A comparison of the components of each cream is presented in Table 1. Each cream was mixed with the standardized components of the electrolyte medium (Schneider \& Fowles, 1978) for initial laboratory evaluation. Once mixed, each cream was examined to determine if each resulted in a suitably viscous medium. One cream, Aquabase, would not mix with the glycol and potassium so it was not tested further.

To meet the aims of the study, 12 volunteers were recruited ( 6 at each site) using word of mouth and flyers posted around campus. Half of the volunteers at each site were postmenopausal women experiencing hot flashes and concomitant perspiration (mean age $=45.67, S D=5.32$ ) and the other half were active young, premenopausal women (mean age $=22.17, S D=1.47$ ). All data were collected during the summer months. Environmental temperatures of the two university settings were comparable (average high: Florida, $89^{\circ} \mathrm{F}$, Tennessee, $86^{\circ} \mathrm{F}$; average low: Florida, $70^{\circ} \mathrm{F}$, Tennessee, $65^{\circ} \mathrm{F}$ ).

The first aim was to determine which of the three creams, when mixed with $100 \mathrm{~g}$ of polyethylene glycol and $0.76 \mathrm{~g}$ potassium chloride (Schneider \& Fowles, 1978), functioned effectively as a medium for use on human skin. For this aim, evaluation of in vivo viscosity of the electrolyte media resulted in elimination of one cream. Potential skin sensitivity to each remaining cream was then assessed. Using a double blind approach, the electrolyte media were identified only as Cream A (Velvachol electrolyte medium) and Cream B (Hydrophilic electrolyte medium). Each volunteer wore two electrodes for a 24-hr period (one electrode filled with approximately $0.4 \mathrm{cc}$ of Cream A and another with Cream B). 
Table 1. Comparison of Unibase Cream Composition with Study Creams

\begin{tabular}{lll}
\hline \hline Unibase & \multicolumn{1}{c}{ Velvachol } & Hydrophilic ointment \\
\hline Water & Water & Water \\
White petroleum & White petroleum & White petroleum \\
$\begin{array}{l}\text { Glycerin } \\
\text { Sodium lauryl sulfate }\end{array}$ & Sodium lauryl sulfate & Sodium lauryl sulfate \\
Propylparaben & Parabens & $\begin{array}{l}\text { Propylparaben } \\
\text { Stearyl alcohol }\end{array}$ \\
& & $\begin{array}{l}\text { Propylene glycol } \\
\text { Mineral oil }\end{array}$ \\
& & Methylparaben \\
& & \\
\hline \hline
\end{tabular}

Meditrace silver/silver chloride 1.5-cm electrodes (Graphic Controls, Buffalo, NY) were used and applied over the sternal area using a standard procedure (Carpenter et al., 1999). Participants were instructed to perform all of their usual daily activities, with the exceptions of bathing or swimming, and return to the research site $24 \mathrm{hr}$ later.

At the end of the 24-hr session, skin integrity, self-report complaints, and electrode integrity were evaluated. Electrodes were removed and assessed for leakage of cream and electrolyte wetness due to perspiration. Next, participants were interviewed to identify any subjective problems, such as itching or burning, encountered during the 24-hr period. Finally, participants' skin integrity was assessed to identify any objective problems due to the cream, such as visible redness or rash.

The second aim of this study was to identify which electrode cream (if any) provided optimal skin conductance in identification of menopausal hot flashes. Participants were asked to wear a set of electrodes containing only either Cream A or Cream B with 24-hr sternal skin conductance monitoring. Skin conductance recordings from the sternal area have been demonstrated as an effective marker for postmenopausal hot flashes (Tataryn et al., 1981) with an $80-95 \%$ concordance rate to other assessment techniques (de Bakker \& Everaerd, 1996; Freedman 1989; Freedman, Woodward \& Norton, 1992). Six participants were monitored using Cream A and 6 participants using Cream B. Cream and geographical site were confounded in this phase because each site tested only one cream, determined through random assignment. However, 1 participant with a self-report of severe hot flashes was initially tested using Cream B. Her skin conductance readings were not readable due to elevated baseline and readings above the monitor limits. After the initial analysis of data from each cream, this participant was subsequently retested using Cream A, providing data for 7 participants with Cream A.

Skin conductance recordings were achieved using the Meditrace electrodes described above (Graphic Controls, Buffalo, NY) and a 0.5 constant voltage circuit (Lykken \& Venables, 1971) built into the front end of a single channel of a Biolog ambulatory recorder (UFI Model 3992/1 SCL, UFI, Morro Bay, CA). Skin conductance was sampled 32 times per second and stored in the Multitrace software for analysis. At the start of their 24-hr session, participants were connected to the monitor, instructed on its use, and then instructed to return to their usual daily activities while wearing the monitor. Participants were instructed to depress an event marker on the Biolog if and when they felt a hot flash occurring. In addition, participants were asked to maintain a diary of their activities (walking, driving, cooking, etc.) while wearing the monitor, recording the time and any activities performed during each hot flash that occurred during the 24-hr session. Participants returned the following day to be disconnected from the monitor. Participants were again interviewed regarding any subjective problems, electrodes were removed and assessed for cream leakage and wetness, and skin integrity was assessed.

Skin conductance data were recorded onto a RAM card, which was downloaded into a personal computer at the conclusion of the monitoring session using customized software (DPS v.1.5, UFI, Morro Bay, CA). Sternal skin conductance changes of $2 \mu$ mho in a 30 -s period were defined as hot flashes. The feasibility and psychometrics of this skin conductance monitor as an objective measure of hot flashes using the Unibase/glycol electrolyte medium have been previously reported (Carpenter et al., 1999).

\section{Results and Discussion}

\section{Skin Integrity and Subjective Complaints}

Leakage of the cream onto the foam ring of the electrode with Cream A was noted in only 1 of 6 subjects, whereas 4 of 6 subjects experienced leakage with Cream B. Leakage is assumed to interrupt electrode effectiveness. Itching and redness were equally reported with each cream. There was only one report of burning (mild, Cream A, Velvachol). No instances of rash were noted with either cream in either phase of testing. However, neither the findings regarding leakage not the one report of burning was significant in statistical analysis.

Descriptive statistics were used for initial analysis of the skin integrity data. Only the occurrence of redness was correlated between Cream A and Cream B, 0.939, $p<.000$, indicating that redness was likely the result of individual skin sensitivity rather than differences in the creams. In subsequent $t$ tests for paired samples, the only significant difference was that of wetness between leads with Cream A or Cream B, $t=3.023, p<.05$.

The creams used in this study have been tested by their manufacturers and are commercially available. It is not likely that the cream should act as a skin irritant or produce discomfort. However, additives to the conductive cream may alter the properties. Although there were no major issues with skin integrity and subjective complaints with either product in this study, further testing in a larger sample is recommended.

\section{Skin Conductance Recordings}

The quality of skin conductance recordings dramatically differed between the two creams (Table 2). Cream A (Velvachol) provided readings that were considered within the normal range previously identified when using this to monitor the Unibase/glycol medium (Carpenter et al., 1999) and reliably identified hot flashes (true positive with minimal false negatives).

Descriptive statistics and $t$ tests for two independent samples were used to analyze the skin conductance data with SPSS 10.0.

Table 2. Electrodermal Skin Conductance Recordings Comparing Creams $A$ and $B$

\begin{tabular}{lcc}
\hline \hline & Cream A & Cream B \\
\hline Baseline & $1-10 \mu$ mhos & $\gg 10 \mu$ mhos \\
True positive hot flashes & 12 & 1 \\
False negative hot flashes & 23 & 114 \\
\hline
\end{tabular}


Table 3. Correlational Analysis of Electrodermal Skin Conductance Recordings

\begin{tabular}{|c|c|c|c|c|c|c|c|}
\hline & Baseline & $\begin{array}{l}\text { Lowest } \\
\text { baseline }\end{array}$ & $\begin{array}{l}\text { Highest } \\
\text { baseline }\end{array}$ & Artifact & $\begin{array}{c}\text { True } \\
+\end{array}$ & $\begin{array}{c}\text { False } \\
-\end{array}$ & $\begin{array}{c}\text { False } \\
+\end{array}$ \\
\hline Baseline & 1.000 & & & & & & \\
\hline $\begin{array}{l}\text { Lowest } \\
\text { baseline }\end{array}$ & $\begin{array}{r}0.728 \\
p<.01\end{array}$ & 1.000 & & & & & \\
\hline $\begin{array}{l}\text { Highest } \\
\text { baseline }\end{array}$ & $\begin{array}{r}0.781 \\
p<.01\end{array}$ & $\begin{array}{c}8.22 \\
p<.01\end{array}$ & 1.000 & & & & \\
\hline Artifact & $\begin{array}{l}0.359 \\
\text { n.s. }\end{array}$ & $\begin{array}{c}-0.113 \\
\text { n.s. }\end{array}$ & $\begin{array}{l}0.303 \\
\text { n.s. }\end{array}$ & 1.000 & & & \\
\hline True + & $\begin{array}{c}-0.475 \\
\text { n.s. }\end{array}$ & $\begin{array}{c}-0.481 \\
\text { n.s. }\end{array}$ & $\begin{array}{c}-0.500 \\
\text { n.s. }\end{array}$ & $\begin{array}{c}-0.084 \\
\text { n.s. }\end{array}$ & 1.000 & & \\
\hline False - & $\begin{array}{r}0.663 \\
p<.05\end{array}$ & $\begin{array}{r}0.652 \\
p<.05\end{array}$ & $\begin{array}{l}0.498 \\
\text { n.s. }\end{array}$ & $\begin{array}{r}0.812 \\
p<.01\end{array}$ & $\begin{array}{c}-0.154 \\
\text { n.s. }\end{array}$ & 1.000 & \\
\hline False + & $\begin{array}{l}0.027 \\
\text { n.s. }\end{array}$ & $\begin{array}{l}0.474 \\
\text { n.s. }\end{array}$ & $\begin{array}{l}0.437 \\
\text { n.s. }\end{array}$ & $\begin{array}{c}-0.163 \\
\text { n.s. }\end{array}$ & $\begin{array}{l}0.525 \\
\text { n.s. }\end{array}$ & $\begin{array}{c}0.091 \\
\text { n.s. }\end{array}$ & 1.000 \\
\hline
\end{tabular}

The correlational analysis is presented in Table 3. As expected, baseline recording, lowest baseline reading, and highest baseline reading were correlated. In addition, baseline recording, lowest baseline reading, and artifact were all significantly correlated with the number of false negative recordings. This correlation could also be expected due to the excessively high recordings Cream B provided. Few false negative hot flashes were recorded using Cream $\mathrm{B}$ due to the considerable difficulty in identifying baseline with that cream.

Independent samples $t$ test was used for further analysis. As indicated in Table 4, significant differences between the creams were found for baseline reading, lowest baseline reading, and highest baseline reading. The number of false negative hot flashes recorded approached significance as well. These findings support the conclusion that Cream A using Velvachol provides an effective electrolyte base medium for skin conductance monitoring.

Further support for the effectiveness of Velvachol was provided by the 1 postmenopausal hot flash subject who used the skin conductance monitor for two 24-hr periods (first with Cream B and later with Cream A). Skin conductance results with Cream B were largely unreadable. As illustrated in Figure 1, the skin conductance recordings were consistently elevated and often above the $25 \mu$ mhos recording range of the monitor. Participant self-report, however, indicated that this subject experienced approximately 10 moderate

Table 4. T Test Analysis of Electrodermal Skin Conductance Recording

\begin{tabular}{lcccc}
\hline \hline & \multicolumn{1}{c}{$t$} & $\begin{array}{c}\text { Sig. } \\
\text { (2-tailed) }\end{array}$ & $\begin{array}{c}\text { Mean } \\
\text { Differences }\end{array}$ \\
\hline Baseline & -7.274 & 11 & .000 & -7.55 \\
Lowest baseline & -4.031 & 11 & .002 & -5.26 \\
Highest baseline & -3.864 & 11 & .003 & -10.14 \\
False - & -2.164 & 11 & .053 & -15.71 \\
False + & -0.521 & 10 & .614 & -0.31 \\
True + & 1.370 & 9 & .204 & 1.46 \\
& & & & \\
\hline
\end{tabular}

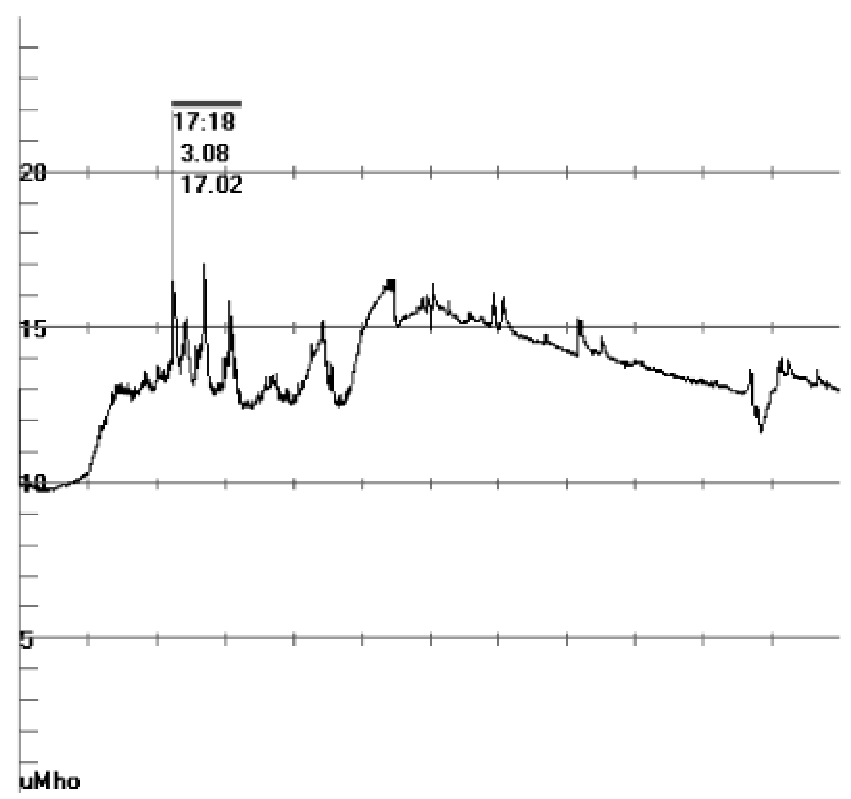

Figure 1. Skin conductance recording of Subject G using Cream B (Hydrophilic ointment) demonstrating elevated baseline and false negative hot flash.

to severe hot flashes during the recording period. After the investigators identified the cream effectiveness difference, this subject was again monitored using Cream A (Velvachol). Results of the repeat testing (see Figure 2) indicated that five true positive hot flashes were identified in this subject and a baseline recording of 1-2 $\mu$ mhos was maintained throughout the recording. The effectiveness of Velvachol as a replacement for Unibase was further supported by the monitoring effectiveness differences in this subject.

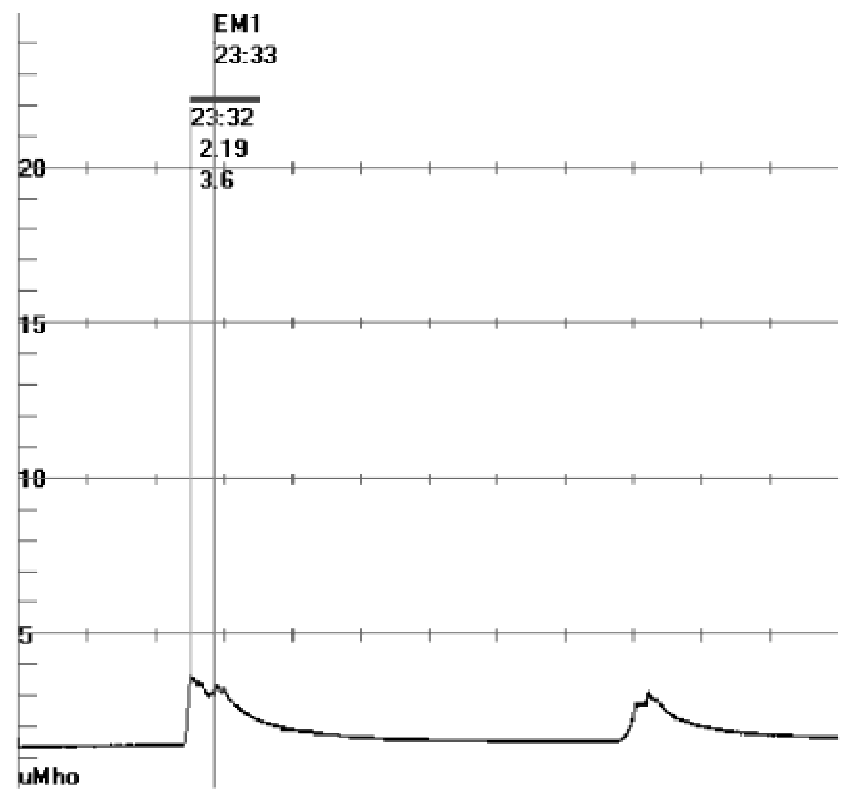

Figure 2. Skin conductance recording of Subject G using Cream A (Velvachol) demonstrating appropriate baseline and true positive hot flash. 
Three base media (Aquabase, Velvachol, and Hydrophilic ointment) and were tested for suitability as a replacement for Unibase in preparation of a skin conductance electrolyte cream. Aquabase did not meet phase I criteria and was not tested further. Of the remaining creams, Velvachol (Cream A) was suitably viscous, did not disrupt skin integrity, and provided quality recordings of skin conductance changes with normal range baselines and overall range normal values. This study indicates that Velvachol is an appropriate substitute for Unibase as an effective nonhydrating electrolyte medium for measurement of sternal electrodermal activity.

Future study should compare the effectiveness of shorter periods of electrode placement with 24-hr placement. In addition, evaluation of Velvachol's effectiveness in recording hot flashes under controlled laboratory conditions and using hot flash induction methods as well as its effectiveness at other sites is needed.

\section{REFERENCES}

Carpenter, J. S., Andrykowski, M. A., Freedman, R. R., \& Munn, R. (1999). Feasibility and psychometrics of an ambulatory hot flash monitoring device. Menopause, 6, 209-215.

de Bakker, I. P. M., \& Everaerd W. (1996). Measurement of menopausal hot flashes: Validation and cross-validation. Maturitas, 25, 87-98.

Freedman, R. R. (1989). Laboratory and ambulatory monitoring of menopausal hot flashes. Psychophysiology, 26, 573-579.

Freedman, R. R., Norton, D., Woodward, S., \& Cornelissen, G. (1995). Core body temperature and circadian rhythm of hot flashes in menopausal women. Journal of Clinical Endocrinology \& Metabolism, 80, 2354-2358.

Freedman, R. R., Woodward, S., \& Norton, D. (1992). Laboratory and ambulatory monitoring of menopausal hot flushes: Comparison of symptomatic and asymptomatic women. Journal of Psychophysiology, 6, 162-166.
Lykken, D. T., \& Venables, P. H. (1971). Direct measurement of skin conductance: A proposal for standardization. Psychophysiology, 8, $656-672$.

Schneider, R., \& Fowles, D. (1978). A convenient, non-hydrating electrolyte medium for the measurement of electrodermal activity. Psychophysiology, 15, 483-486.

Tataryn, I. V., Lomax, P., Meldrum, D. R., Bajorek, J. G., Chesarek, W., \& Judd, H. L. (1981). Objective techniques for the assessment of postmenopausal hot flashes. Obstetrics and Gynecology, 57, 340-344.

(Received March 7, 2000; Accepted November 14, 2001) 